\title{
Techniques for completing Colpotomy without Uterine Manipulator in a Total Laparoscopic Hysterectomy
}

\author{
Shazia Khan ${ }^{1} \odot$, Kuan-Gen Huang ${ }^{2}$, Ala U'wais ${ }^{3}$
}

\begin{abstract}
Aim: To describe techniques used in our setup as an aide to completion of colpotomy in total laparoscopic hysterectomy (TLH), in situations where usage of a uterine manipulator is not feasible.

Background: Over the years, various uterine manipulators have flooded the markets to make the step of colpotomy easier. But there are certain conditions that render the usage of manipulators impossible like narrow vagina, virgin women, retracted or pulled up cervix, and non availability of advance manipulators due to financial constraints. Performing TLH in these conditions is challenging, and certain technical innovations aid in ensuring safe completion of the surgery.

Technique: We describe the techniques utilizing a gauze on the sponge holder to delineate the cervicovaginal junction. The anterior approach, posterior approach, approach through uterosacral ligament, and cardinal ligament are described for completion of colpotomy.

Conclusion: In situations where the application of vaginal manipulators is not feasible, certain technical modifications and varying approaches to the cervicovaginal junction can aid in the successful completion of colpotomy in TLH.

Clinical significance: Vaginal manipulator is a useful instrument in laparoscopic hysterectomy. It manipulates the uterus in cephalad, lateral, and anteroposterior directions, making surgical steps in laparoscopic hysterectomy easier and quicker to perform. Its vital role in safely delineating anatomical landmarks like a uterocervical junction, uterosacral ligaments, and ureter cannot be denied. However, there are certain circumstances where the vaginal route is not accessible, and insertion of a uterine manipulator becomes impossible. In these circumstances, the surgeon should know the other options that help in opening the cervicovaginal junction so that he can complete the surgery safely. Our techniques provide an aid for such difficult situations.

Keywords: Colpotomy; Total laparoscopic hysterectomy, Uterine manipulator.

Journal of South Asian Federation of Obstetrics and Gynaecology (2021): 10.5005/jp-journals-10006-1853
\end{abstract}

\section{INTRODUCTION}

Before Harry Reich first performed laparoscopic total hysterectomy in January 1988 and published his method in 1989, abdominal and vaginal routes were the most common routes for total hysterectomy. The past three decades have seen a rising trend in total laparoscopic hysterectomies (TLH) largely due to the established benefits of lower postoperative morbidity, early return to normal activities, better cosmesis, lower costs, and improved quality of health care. Enthusiastic pioneers have since then reviewed, analyzed, and come up with several modifications of standard steps of laparoscopic hysterectomy. ${ }^{1}$

Harry Reich had described TLH using Wolff's vaginal manipulator/ delineator initially. ${ }^{2}$ Over the years since that, various uterine manipulators have flooded the markets to make the step of colpotomy easier. But there are certain conditions that render the usage of manipulators impossible like narrow vagina, virgin women, retracted or pulled up cervix, and nonavailability of advance manipulators due to financial constraints. Performing TLH in these conditions is challenging, and certain technical innovations aid in ensuring a safe completion of the surgery. ${ }^{3}$ We aim to describe a few such techniques routinely practiced in our setup as an aide for successful TLH.

\section{Colpotomy Techniques without Uterine Manipulator}

\section{Approach for Anterior Colpotomy}

This approach can be used when the vaginal manipulator cannot be applied in cases such as the narrow vagina, cervical stenosis, or even in
${ }^{1}$ Department of Obstetrics and Gynecology, INHS Asvini, Mumbai, Maharashtra, India

${ }^{2}$ Department of Obstetrics and Gynecology, Linkou Chang Gung Memorial Hospital, Taoyuan, Taiwan, China

${ }^{3}$ Department of Obstetrics and Gynecology, Mutah University, Karak, Jordan

Corresponding Author: Kuan-Gen Huang, Department of Obstetrics and Gynecology, Linkou Chang Gung Memorial Hospital, Taoyuan, Taiwan, China, e-mail: kghuang@ms57.hinet.net

How to cite this article: Khan S, Huang K G, U'wais A. Techniques for Completing Colpotomy without Uterine Manipulator in a Total Laparoscopic Hysterectomy. J South Asian Feder Obst Gynae 2021;13(1):74-76.

Source of support: Nil

Conflict of interest: None

virgin women. Often in these situations, vaginal access is still possible using simple narrow instruments only, such as sponge forceps.

The surgeon can delineate the anterior fornix by applying a "swab on a stick," i.e., a sponge forceps with a $4 \times 4$ gauze held folded at its tip, in the anterior vaginal fornix, and pushing the fornix cephalad and ventrally. This maneuver makes the apex of the anterior vaginal wall bulge out and can be seen clearly (Fig. 1A). This step can also be used to dissect the bladder against the anterior vaginal wall and separate the cervix anteriorly from the vagina by using the monopolar hook (Fig. 1B).

(c) Jaypee Brothers Medical Publishers. 2021 Open Access This article is distributed under the terms of the Creative Commons Attribution 4.0 International License (https://creativecommons.org/licenses/by-nc/4.0/), which permits unrestricted use, distribution, and non-commercial reproduction in any medium, provided you give appropriate credit to the original author(s) and the source, provide a link to the Creative Commons license, and indicate if changes were made. The Creative Commons Public Domain Dedication waiver (http://creativecommons.org/publicdomain/zero/1.0/) applies to the data made available in this article, unless otherwise stated. 


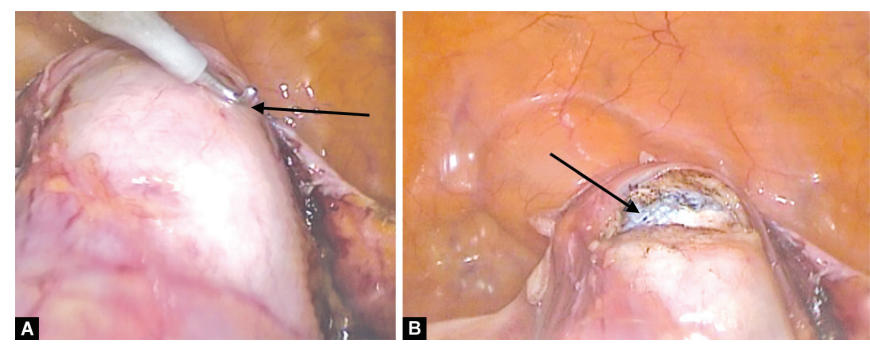

Figs. 1A and B: (A) Delineation of the anterior fornix using a swab; (B) The swab appears after cutting the apex of the anterior vaginal wall

\section{Approach for Posterior Colpotomy}

Posterior colpotomy appears a good option to start with when separating the cervix from the vaginal wall. Some centers recommend it as the better method of starting colpotomy. But, after opening the posterior fornix, it is difficult to grasp the cervix and pull it outside the incision. The insertion of the uterosacral ligament at the posterolateral aspect of the cervix prevents its eversion backward. Thus, if the surgeon had decided to start with posterior colpotomy, he should divide the uterosacral ligament bilaterally before attempting to open the posterior fornix. This will help in everting the posterior lip of the cervix outside the posterior colpotomy incision easily. The posterior fornix can again be delineated using sponge forceps and $4 \times 4 \mathrm{~cm}$ gauze (swab on stick), as described in the anterior colpotomy approach (Fig. 2).

\section{Left Uterosacral Approach}

This approach has advantages over the other suggested approaches for many reasons. The uterosacral ligament is a clear anatomical landmark. It is a taut structure and does not need vaginal aid for identification. The ureters are relatively far from the incision site, especially while considering lateral thermal damage. We suggest the left side uterosacral ligament because the surgeon usually stands on the left side, which makes it more ergonomical and easily accessible. In addition, the rectum physiologically deviates slightly to the right side.

To identify the uterosacral ligament clearly, the surgeon can push the cervix anteriorly (ventrally) by pushing the posterior aspect of the isthmus forward. This step will open up the pouch of Douglas and facilitate the division of the left uterosacral ligament. Then, the ligament is electrocoagulated with an energy device, at its insertion to the uterus and cut. Continuing to cut in the same plane toward the vagina will open the vaginal wall at the level of the cervicovaginal junction. Then, the incision is extended posteriorly and anteriorly. The cervix can then be grasped and pulled upward (cephalad) using a single tooth forceps so that the surgeon can coagulate and cut along the cervical edge to complete the colpotomy (Fig. 3).

\section{Cardinal Ligament Approach}

Opening the lateral fornix after cutting the cardinal ligament is considered a feasible approach and can be performed without a vaginal assistant. However, the surgeon must keep in mind the proximity of the ureter and the risk to it of both a direct injury and a thermal damage. Thus, the surgeon must identify the ureter's course before coagulating and cutting the cardinal ligament. The division of the cardinal ligament should be as perpendicular as possible to its insertion to the cervix, keeping in mind that the bladder has already been dissected and pushed below the cutting

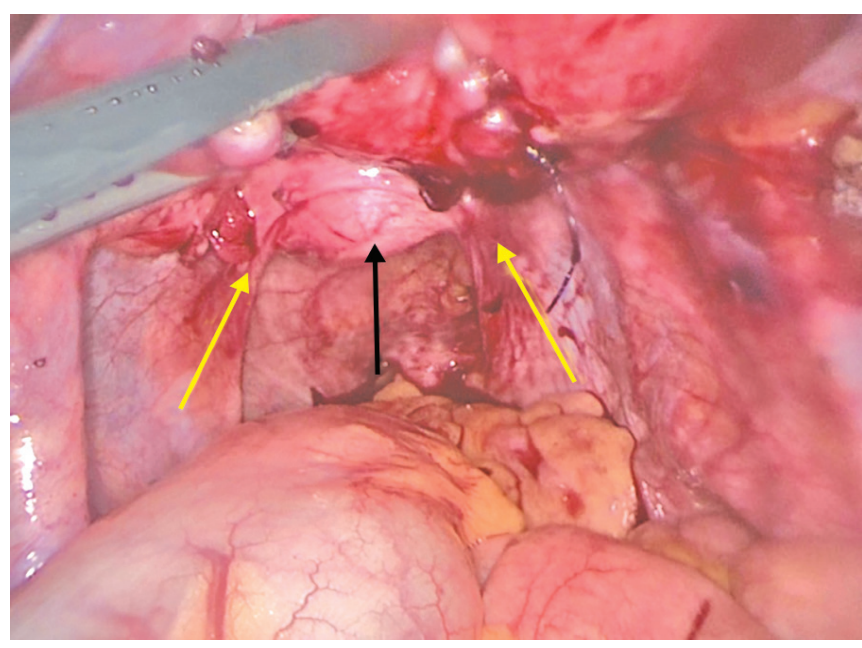

Fig. 2: Black arrow points to the delineated posterior fornix. Yellow arrows point to the uterosacral insertion

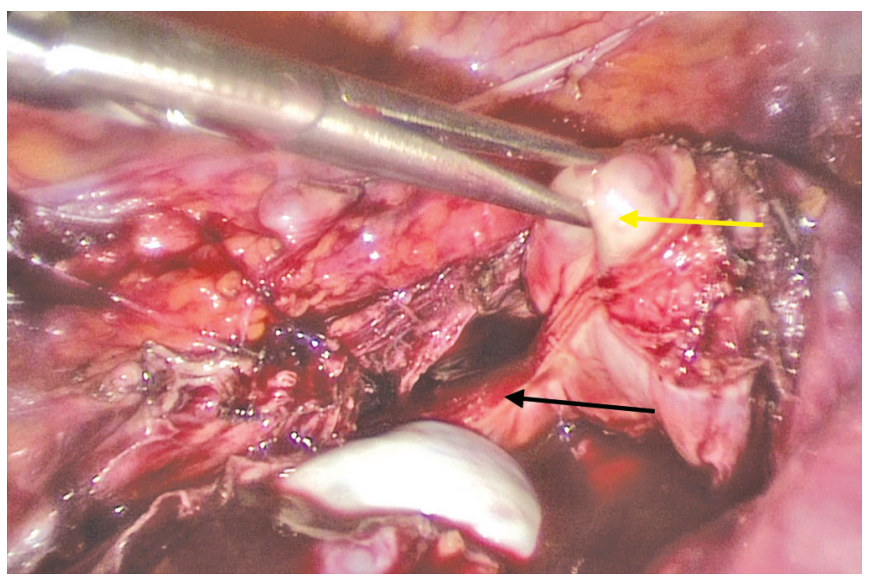

Fig. 3: Black arrow points to the cervicovaginal junction. Yellow arrow points to the cervix

level. After dividing the ligament, the vaginal wall is opened by cutting at the insertion of the cardinal ligament obliquely. Then, the colpotomy is extended, and the vaginal wall is cut at the level of the cervicovaginal junction.

\section{Discussion and Clinical Significance}

The advent of minimally invasive approaches to hysterectomy has seen varied modifications of techniques while retaining the basic principles of the open surgery. The recent advances in instrumentation and higher energy devices in minimally invasive technology along with favorable postsurgical outcomes have led to TLH being established as the procedure of choice for various uterine and adnexal pathologies by gynecological endoscopists. The various approaches of laparoscopic hysterectomy are reliant on the use of uterine/vaginal manipulators. ${ }^{4}$ Nevertheless, the literature review reveals limited reports on completion of colpotomy step of TLH approach without utilizing either vaginal or uterine manipulator as an accessory. Our approaches demonstrate a safe, efficient, and an easy to learn technique to perform TLH without the use of any uterovaginal manipulator. This approach can help to reduce operative time by eliminating the use of an extra assistant to 
handle uterine manipulator and reducing blood loss by minimizing any traumatic movement through the vagina or uterus. ${ }^{5}$ Moreover, the use of simple readily available supplements like sponge holder and tenaculum forceps makes the above-mentioned techniques cheap and easily reproducible in low resource settings.

\section{CONCLUSION}

Vaginal manipulator is a useful instrument in laparoscopic hysterectomy. It manipulates the uterus in cephalad, lateral, and anteroposterior directions, making surgical steps in laparoscopic hysterectomy easier and quicker to perform. Its vital role in safely delineating anatomical landmarks like uterocervical junction, uterosacral ligaments, and ureter cannot be denied. However, there are certain circumstances where the vaginal route is not accessible, and insertion of a uterine manipulator becomes impossible. In these circumstances, the surgeon should know the other options that help in opening the vagina at its junction to the cervix so that he can complete the surgery safely. Our techniques provide an aid for such difficult situations.

\section{ORCID}

Shazia Khan 1 https://orcid.org/0000-0001-6284-4311

\section{References}

1. The American College of Obstetricians and Gynecologists (ACOG). Committee Opinion No 701: choosing the route of hysterectomy for benign disease. Obstet Gynecol 2017;129(6):e155-e159. DOI: 10.1097/ AOG. 0000000000002112.

2. Reich H, McGlynn F, Sekel L. Total laparoscopic hysterectomy Gynaecol Endosc 1993;2: 59-63.

3. Van den Haak L, Alleblas C, Nieboer TE, et al. Efficacy and safety of uterine manipulators in laparoscopic surgery: a review. Arch Gynecol Obstet 2015;292(5): 1003-1011. DOI: 10.1007/s00404-015-3727-9.

4. Popa A, Copaescu C, Horhoianu V. Laparoscopic total hysterectomy still not routinely chosen: operative description and available instruments. J Med Life 2019;12(3): 301-307. DOI: 10.25122/jml-2019-0051.

5. Macciò A, Madeddu C, Kotsonis P, et al. Feasibility and safety of total laparoscopic hysterectomy for huge uteri without the use of uterine manipulator: description of emblematic cases. Gynecol Surg 2018; 15(1): 6. DOI: 10.1186/s10397-018-1037-5. 